\title{
Cherenkov Telescope Array Data Management
}

\author{
G. Lamanna ${ }^{* a}$, L.A. Antonelli ${ }^{b, c}$, J.L. Contreras $^{d}$, J. Knödlseder $^{e}$, K. Kosack ${ }^{f}, \mathbf{N}$.

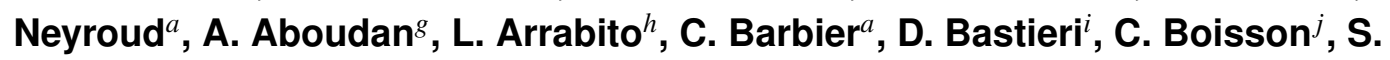
Brau-Noguée $^{e}$ J. Bregeon ${ }^{h}$, A. Bulgarelli ${ }^{g}$, A. Carosi ${ }^{b, c}$, A. Costa ${ }^{k}$, G. De Cesare ${ }^{g}, \mathbf{R}$. de los Reyes ${ }^{l}$, V. Fioretti ${ }^{g}$, S. Gallozzi ${ }^{b}$, J. Jacquemier ${ }^{a}$, B. Khelifi ${ }^{m}$, J. Kocot ${ }^{n}$, S. Lombardi $^{b, c}$, F. Lucarelli ${ }^{b, c}$, E. Lyard ${ }^{o}$, G. Maier ${ }^{p}$, P. Massimino ${ }^{k}$, J. P. Osborne ${ }^{q}$, M. Perri $^{b, c}$, J. Rico ${ }^{r}$, D. A. Sanchez ${ }^{a}$, K. Satalecka ${ }^{d}$, H. Siejkowski ${ }^{n}$, T. Stolarczyk ${ }^{f}$, T. Szepieniec $^{n}$, V. Testa ${ }^{b}$, R. Walter ${ }^{o}$, J.E. Ward ${ }^{r}$ and A. Zoli ${ }^{g}$ for the CTA consortium ${ }^{a}$ LAPP, Université de Savoie Mont-Blanc, CNRS/IN2P3, F-74941 Annecy-le-Vieux, France ${ }^{b}$ INAF - Osservatorio Astronomico di Roma, Via Frascati, 33, I-00040 Monte Porzio Catone ${ }^{c}$ ASI Science Data Center, Via del Politecnico, s.n.c., I-00133 Rome, Italy

${ }^{d}$ Grupo de Altas Energías, Universidad Complutense de Madrid, Spain

${ }^{e}$ IRAP, 9, avenue du Colonel Roche BP 44346 - 31028 Toulouse, France

${ }^{f}$ CEA Saclay - Irfu/SAp - Orme des merisiers, Bât. 709 - 91191 Gif-sur-Yvette Cedex, France

${ }^{g}$ INAF/IASF, Via Piero Gobetti 101, 40129 Bologna, Italy

${ }^{h}$ LUPM, CNRS-IN2P3 Place Eugéne Bataillon - CC 7234095 Montpellier, France

${ }^{i}$ Dipartimento di Fisica, Universita degli studi di Padova, Italy

${ }^{j}$ Observatoire de Paris, LUTH, CNRS, Université Paris Diderot, Paris, France

${ }^{k}$ INAF - Osservatorio Astrofisico di Catania, Via S.Sofia 78, 95123 Catania, Italy

${ }^{l}$ Max-Planck-Institut für Kernphysik Saupfercheckweg 1, 69117 Heidelberg, Germany

${ }^{m}$ APC, IN2P3/CNRS, Université Paris-Diderot, Paris, France

${ }^{n}$ Academic Computer Centre CYFRONET AGH, Kraków, Poland

${ }^{\circ}$ ISDC, University of Geneva, Switzerland

${ }^{p}$ Deutsches Elektronen-Synchrotron Platanenallee 6, Zeuthen, Germany

${ }^{q}$ Dept. of Physics \& Astronomy, University of Leicester, Leicester LE1 7RH, GB

${ }^{r}$ IFAE, Edifici Cn. Universitat Autonoma de Barcelona 08193 Bellaterra (Barcelona) Spain

E-mail: giovanni.lamanna@lapp.in2p3.fr

Very High Energy gamma-ray astronomy with the Cherenkov Telescope Array (CTA) is evolving towards the model of a public observatory. Handling, processing and archiving the large amount of data generated by the CTA instruments and delivering scientific products are some of the challenges in designing the CTA Data Management. The participation of scientists from within CTA Consortium and from the greater worldwide scientific community necessitates a sophisticated scientific analysis system capable of providing unified and efficient user access to data, software and computing resources. Data Management is designed to respond to three main issues: (i) the treatment and flow of data from remote telescopes; (ii) "big-data" archiving and processing; (iii) and open data access. In this communication the overall technical design of the CTA Data Management, current major developments and prototypes are presented.

The 34th International Cosmic Ray Conference,

30 July- 6 August, 2015

The Hague, The Netherlands 


\section{Overall Concept}

CTA is a new observatory for very high-energy (VHE) gamma rays [1]. The Data Management (DATA) project of CTA concerns all major components for both data-flow administration and the scientific data production and analysis for CTA. The main scope of the project is the design of the CTA Science Data Centre (SDC), which is in charge of the off-site handling of data reduction, Monte Carlo (MC) simulations, data archiving and data dissemination. The remote (e.g. intercontinental) transmission of data from CTA sites to the CTA archive is one of the key services that the SDC administers at both ends: off and on the CTA site. The development and provision of software and middle-ware services for dissemination including observation proposal handling is a task that DATA guarantees to be interfaced with the Operation Centre. The services and components that DATA is in charge of at the CTA sites (Telescope Array Control Centres) include: the execution of on-site scientific data reduction pipelines, the real-time analysis, the on-site temporary archive system as well as the data quality monitoring.

\section{Summary of Design}

The DATA design is inspired by the lessons learned from current and past Imaging Atmospheric Cherenkov Telescopes, from CTA telescopes prototype, from existing astronomical observatories, and finally from the technical know-how of major computing / data centres and einfrastructures that serve large international projects. Figure 1 depicts the main path and rate of data within the CTA Observatory (CTAO). On each CTA site, the data rates are based on the event rates from Cherenkov and night-sky-background triggers registered by the telescope array. The rates depend strongly on: the number and type of telescopes in the two arrays $(\sim 130$ in the current assumptions), the number of pixels per camera, the nominal trigger rates, the length (in time) of the pixel readout windows, the number of samples per unit time, and the number of bytes recorded per sample. Some pre-processing and filtering of stereoscopic Cherenkov events will affect the nominal data rates, which will result in $5.4 \mathrm{~GB} / \mathrm{s}$ for CTA south (with $\sim 100$ telescopes) and 3.2 $\mathrm{GB} / \mathrm{s}$ from CTA north (with $\sim 30$ telescopes). They also include $20 \%$ calibration data and $10 \mathrm{MB} / \mathrm{s}$ of device monitoring and control data for each site, for a resulting total data rate of about $8.6 \mathrm{~GB} / \mathrm{s}$. The remote connection to the CTA site candidates must satisfy the bandwidth capacity of $1 \mathrm{~Gb} / \mathrm{s}$, which makes the issue of the exported data size critical. During the construction phase, DATA will develop a data volume reduction system that cuts the nominal data rate by a factor of 10 , thus implying an output rate of only $0.32 \mathrm{~GB} / \mathrm{s}$ and $0.54 \mathrm{~GB} / \mathrm{s}$ data from CTA north and south respectively. These data rates are valid over an annual duty cycle of $1314 \mathrm{~h}$ and they will correspond to an equivalent and continuous data volume flux of $0.38 \mathrm{~Gb} / \mathrm{s}$ and $0.65 \mathrm{~Gb} / \mathrm{s}$, well manageable (with respect to the latency requirements) with a local temporary storage unit on the CTA sites, and a 1 $\mathrm{Gb} / \mathrm{s}$ effective network for off-site data export. Current perspectives for the availability of $10 \mathrm{~Gb} / \mathrm{s}$ network from candidate CTA sites exist.

The exported data are received by the ingestion unit of the CTA Archive system, which is operated (together with all main work-flow management services) by a dedicated Observatory Data

\footnotetext{
*Speaker.

${ }^{\dagger}$ Full consortium list at http://cta-observatory.org
} 


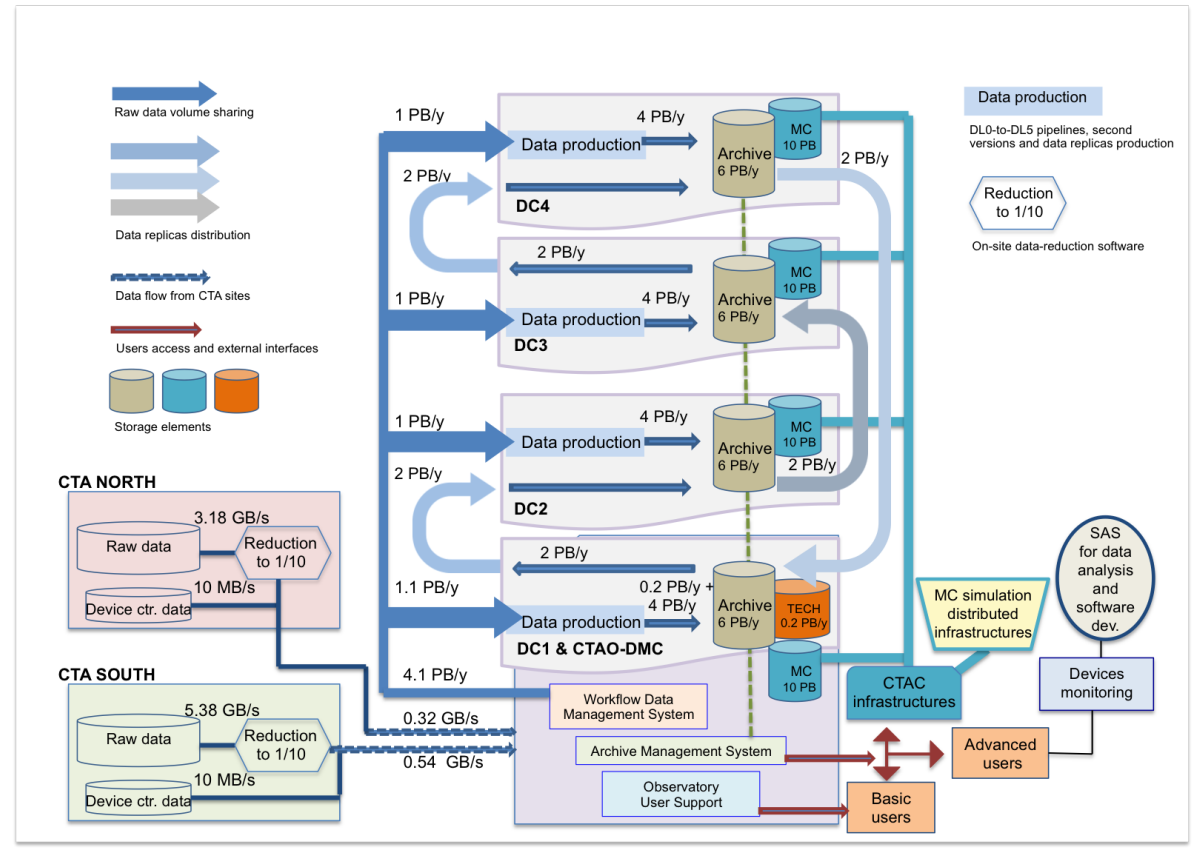

Figure 1: CTAO data volume management. Raw data and device control data are transferred from CTA sites to the CTAO Data Management Centre along the network. Data are then distributed over four data-centres participating in the processing and archive of data. The total data volume, including replicas, is managed by the four data-centres sharing the CTAO storage.

Management Centre (CTAO-DMC). The proposed CTAO computing model is built upon a Distributed Computing Infrastructure (DCI) approach, in which a limited number of first-class dataand-computing centres share the workload of archiving and processing the CTA data. The baseline of DCI model adopted to estimate the work and investment distribution is made of four centres (from DC1 to DC4), equally sharing the CTAO data workload (Figure 1). One out of these four centres also hosts the CTAO-DMC. The CTAO-DMC plus the four DCs correspond together to the proposed implementation of the CTA Science Data Centre. The CTAO-DMC simultaneously guarantees: (i) the orchestration of the relay-mode activities among the four centres, while centrally managing the database of the CTAO archive; (ii) all interface services with the users, providing tools to the Observatory User Support group and to advanced users for archive queries and data processing or to access technical data for devices monitoring purposes as well as User Support; (iii) the integration of the Scientific Analysis System (SAS) with the CTAO and the CTA Consortium (CTAC) infrastructures, which are also DCIs and are used currently for MC simulation production (through the existing CTA Computing Grid infrastructure - CTACG). Each data-centre will permanently archive one half of the full CTA data: it receives $25 \%$ of raw data to be processed and will play the role of back-up centre archiving the replica of another $25 \%$ of the data, coming form another centre. The geographical location of these centres is not specified, and from the technical point of view any place where a qualified (such as WLCG Tier1-like) data centre is willing to contribute to the implementation of the model (including in the CTA array host countries) may be considered. The total volume to be managed by the CTAO Archive is of the order of $27 \mathrm{~PB} / \mathrm{year}$, when all data-set versions and backup replicas are considered. This will correspond to a permanent 
archive of the order of more than 400 PB in 2031.

The computing needs are less critical: peak values of less than $10^{4} \mathrm{CPU}$ cores are expected for the annual data processing. The CTA data processing is based on several levels of processing: the MC simulation pipeline, the low-level data processing pipelines, the high-level science software tools and the Virtual Observatory (VO) data access services. The speed and performance requirements of the design of the data processing pipelines necessitate a high level of parallelization. This will imply a large effort to reducing the I/O speed and CPU time required. This is particularly relevant in the case of real-time or on-site analysis, which initially will use similar software to that used off-site, but with more strict requirements on speed and looser requirements on accuracy. However, the adoption of new computing architectures (e.g. GPGPU, ARM processor) to reduce the cost and improve the performance of the data streaming and accelerate the processing, are not excluded in the current design.

MC simulations are required to characterize the performance and response of the instrument to Cherenkov light emitted in extensive air showers. Simulations are also necessary for the development of reconstruction and analysis tools. Therefore a large quantity of simulated data (20 PB) will be produced prior to the operations phase (during the construction phase) and permanently archived. MC processing is also envisioned during the operation phase to produce up-to-date Instrument Response Functions (IRF), and for validation of new algorithms and software versions. The format of simulated data and software for reconstruction and analysis are identical to those applied to observation data (with some extra information attached, corresponding to simulated parameters).

Off-site, in each $\mathrm{DC}_{i}$ data center, the data production consists of a series of processing steps that transform archived (reduced) raw data DL0 (Data Level 0) to calibrated camera data (DL1), then to reconstructed shower parameters such as energy, direction, and particle ID (DL2), and finally to high-level observatory products comprised of selected gamma-like events, instrument response tables, and housekeeping data (DL3). DL3 data will have a total volume of about $2 \%$ of the DL0 data volume and guaranteed access will be provided in the CTA archive to basic users (e.g. Guest Observers and Archive Users). The science tools are then used either automatically or by users to produce DL4 (e.g. spectra, sky-maps). Finally (DL5) legacy observatory data, such as CTA survey sky maps or the CTA source catalog will be produced. When all replicas and versions are considered, according to user analysis requirements and archive optimization, the data processing and archiving processes generate $27 \mathrm{~PB} /$ year data out of incoming reduced DL0 4 PB data per year (Figure 1$)$. The CTAO computing model makes use of disk $(\sim 20 \%)$ and tape storage $(\sim 80 \%)$ to guarantee effective storage, access and throughput of all data, while trying to reduce the associated costs.

Another big challenge for the management of data by the CTAO is the open access to the CTA data. To operate as an open observatory, a minimum set of services and tools are needed to support the scientific users of CTA. These services are intended to be mostly web-oriented and consist of: electronic support services to help Guest Observers in writing and submitting a proposal to CTA in response to an Announcement of Opportunity for observing time; user interfaces to follow the status of an observation, including the scheduling, the data acquisition, the data processing, the data distribution and the ingestion of the data in the public archive after the end of the proprietary period; and finally services for downloading the processed data (DL3) as well as the software tools 
that are necessary for scientific analysis. Web-based information about the data and the analysis software, including user manuals, cook books, etc. will also be available. Archive Users will browse the archive to access and retrieve CTA data of interest selecting events based on specific criteria (source location, observation time interval, observation condition criteria, energy range etc.). CTA basic users will analyse the data will be analysed on their own computing.

DATA will ensure the integration of CTA high-level data (DL4-to-DL5) within the Virtual Observatory (VO) infrastructure, by adopting and extending the VO data model standards suitable for the description of gamma-ray data. High-energy data at this level have never before been available to the VO community, and this represents a major step toward unifying the data products from all high-energy experiments. Current astronomical metadata standards and VHE gamma-ray data conventions have been studied for this purpose via a close working relationship between both VHE and VO scientists. The extension of standard VO semantic models will allow compatibility with a large range of existing tools and infrastructures for data discovery, visualization, and processing. Detailed Characterization Data Model fields will be completed for high-level products (images, spectra, light curves) and also for event lists and (Instrument Response Functions) IRFs, allowing scientists from other backgrounds to discover and manipulate high-level CTA data products without requiring specialized CTA tools for all operations. The final goal is to integrate CTAO data in astronomical multi-wavelength data archives where scientist will be able to combine them together in a single analysis with data from other facilities.

The size and the world-wide scope of the CTA consortium, along with the desire of CTAC advanced users to access the full archive and to manage more complex analysis work-flows, demands the implementation of services to operate a common scientific analysis platform. In this respect an important baseline of the IC-infrastructure solution for data access is the CTA Scientific Gateway: a web-based community-specific set of tools, applications, and data collections that are integrated together via a web portal, providing access to resources and services from a distributed computing infrastructure. The Gateway aims at supporting work-flow handling, virtualization of hardware, visualization as well as resource discovery, job execution, access to data collections, and applications and tools for data analysis. Furthermore the Gateway may even potentially host all monitoring services of data operation as well as some remote control or monitoring applications for instruments and devices when applicable. The continuous and cooperative software development within the CTAC requires some consortium shared services such as a software repository, development tools, version track services and software validation test benches. Most are currently implemented and already in use in CTA, and will evolve in the future into a single web-oriented global platform of services.

Access to the development services, Gateway, and other CTA web resources will be based on each user's profile and category (e.g. basic, advanced users, managers, collaboration users, etc). For such a purpose an Authentication and Authorization infrastructure is under development and will be applied to extend the use of the CTA Gateway to any user tuned according to their own role and/or access rights.

\section{Main developments and prototyping}

Currently the CTAC organizes the DATA development activities around five main basic com- 
ponents: (i) data model, (ii) archives, (iii) pipelines, (iv) observer access and (v) IC-infrastructures.

\subsection{Data Model}

In the context of the development of a global Data Model for CTA, some prototyping work has been conducted around the application of Compressed FITs format (CFITS) as a file format for Low Level Data, resulting in a viable and cost-effective solution. For higher level camera data a flexible format is looked for. Three formats are under consideration and prototyped: one based on the Google Protocol Buffers specification, a specific eventio format developed for the HESS experiment, and a stream data format based on satellite missions: packetLib. Packetlib, is a software library that manages complex data layouts described with XML files, providing introspection, having a strong memory management and performing a non linear decoding: this, coupled with the Consultative Committee for Space Data Systems (CCSDS) space packets standard enables a fast and on-the-fly data identification, access and routing. For the high level data comparative studies are conducted among FITS, ROOT and HDF5 file formats.

\subsection{Pipelines}

Pipelines refers to all software components necessary to process real and simulated raw data and produce the final end products needed for science analysis, along with any associated quality monitoring and technical data. A pipeline is defined as a sequence of data processing steps that are applied to data to achieve a high-level goal. The logical design of data reconstruction and analysis pipelines is well understood, it relies on the software in use in current experiments such as HESS, MAGIC and VERITAS, complemented by recent explorative developments from precursors such as FACT and ASTRI. Main prototyping activities were around the evaluation of software frameworks based on $\mathrm{C} / \mathrm{C}++$ languages, Python, Hadoop using modern MapReduce computing method. Further comparative studies concerned: adapting FITS data format to Hadoop files management methods, combining Python and $\mathrm{C}++$ for inter-process communication in the context of the real-time data streaming [2] with FPGA and GPU hardware accelerators.

\subsection{Archives}

The CTA Archives development are inspired by the lessons learned by operating astronomical Observatory. The global architecture has been designed based on the ISO OAIS Reference Model. Main prototyping activities have been dedicated to explore the new database solutions as a function of data type, volume and expected rate and type of archive queries, e.g. the open-source, highperformance MongoDB prototype both for housekeeping information and scientific raw data.

\subsection{Observer Access}

Main prototyping activities are around the analysis software for open access ctools: a set of analysis executables that is largely inspired from HEASARC's ftools and Fermi's Science Tools, allowing the assembly of modular workflows for CTA data analyses. The ctools comprise so far executables to simulate CTA event lists, to select and bin the data, to perform a maximum likelihood analysis, and to create sky images (see also [3]). 


\subsection{Information and Computing infrastructures}

The majority of the computing resources for the CTA massive MC simulation production and analysis are linked to each other with the Grid e-infrastructures and they have allowed DATA to evaluate the Grid approach through a dedicated pathfinder initiative: CTACG. Among the different prototyping activities within CTACG, the DIRAC (Distributed Infrastructure with Remote Agent Control) WMS prototype was intensively tested and evaluated as a solution to handle the MC production. DIRAC was identified as useful and appropriate for the following purposes: (i) Production job handling: e.g. for pipelines (Monte-Carlo Production, Data reduction pipeline, Onsite Reconstruction and Analysis); (ii) User analysis job handling and data management [4]. Some prototyping activities were conducted in order to build up an SAS through the web-base Science Gateway for CTA. Two complementary solutions based on the same underlying portal middleware LifeRay were developed with different aims: the first one integrating existing CTA Applications in a specific InSilicoLab framework, the second one with a specific focus on Authentication, Authorization and Single Sign On, with an architecture based on WS- PGRADE/gUSE framework for integration of applications, and more recently enriched with an interactive desktop environment named ACID. A third prototype, compliant with the VO, has been developed based on the Django framework. DATA is currently building a final Gateway prototyping merging the complementary services provided by the these three examples.

\section{Organization}

The organization of the activities within DATA is structured around the evolution of the overall CTA project, the complementary of the CTAC and CTAO roles and their respective internal managements, and the proposed computing and functional models. In Figure 2 an overview of the logical implementation of the DATA baseline design is represented. At the same time some growing operational activities are managed by DATA: e.g. the software development services centrally managed at the computing centre CCIN2P3 and supported by a dedicated international "CTA support group"; the DCI-Grid resources technical coordination for massive MC production (e.g. CTACG), including more than twenty computing centres distributed in several countries (France, Germany, Poland, Italy, Spain and others). During the construction phase, the DATA project organization will evolve to take into account the requirements settled on by the test and pre-production operation phases of an increasing number of DATA products. The CTAC will organize itself in "competence centres" such as specific and continuous software development groups or technical and auxiliary devices monitoring groups (see Figure 2). In the construction phase the same competence groups will be in charge of bringing in operation and support their products. During the operation phase some key experts within the CTAO will have the commitment to guarantee the operation and maintenance of any piece of software and DATA services, while the competence centres will be the CTAC instances, which will guarantee the software and services upgrading according to the contingent needs. The scientific and operative link between CTAC and CTAO in DATA will be represented by the shared scientific analysis platform. 


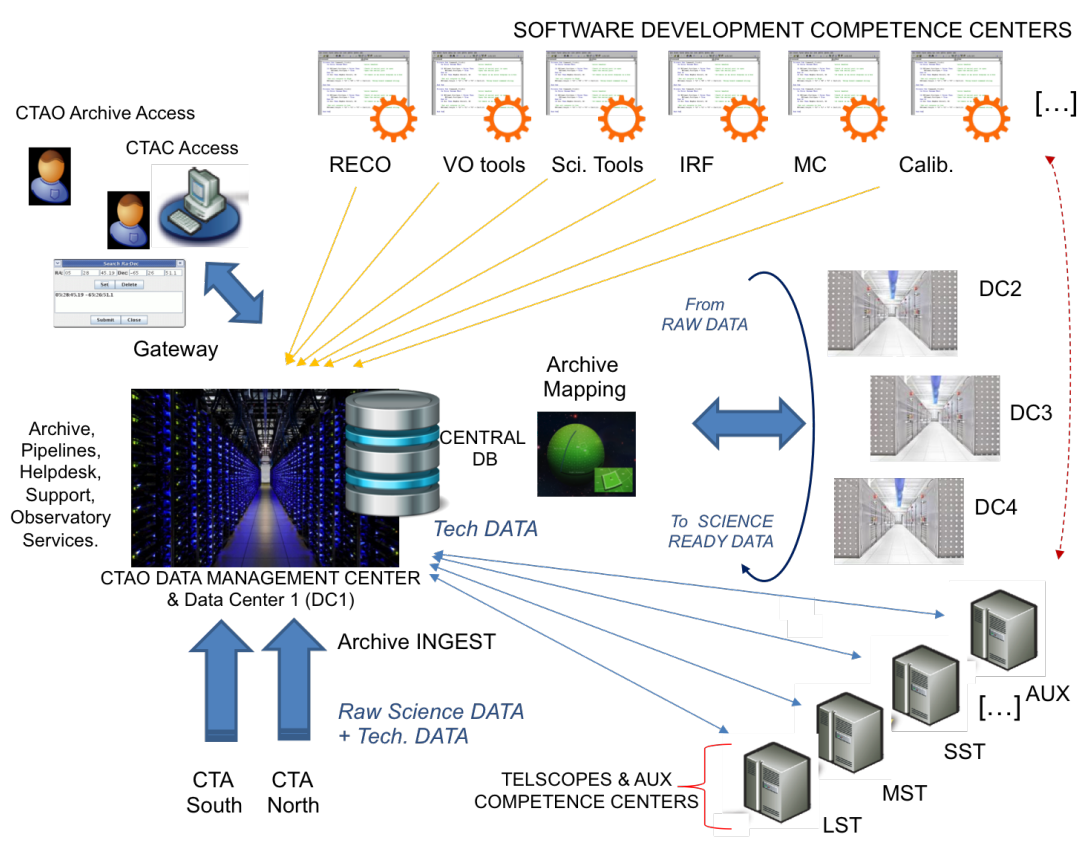

Figure 2: Logical diagram of the CTA Observatory functional Units. The CTAC competence centres guarantee the software and DATA services upgrading, while the CTAO will run them into the CTAO Data Management Centre. The "telescopes and auxiliaries competence centres" are those expert groups in any specific antenna, camera, device needing access to the Tech data (archived in DC1 and made available by the CTAO) for off-site monitoring purpose. The link with the Calibration competence centres will guarantee that all major changes in the software, which depend on Tech data are taken into account during the upgrading.

\section{Conclusions}

A technical design of the complete data life cycle and management for the CTA observatory has been finalized. A set of solutions and a series of prototypes have been proposed to organize the Science Data Center of the CTA observatory. In the next years, entering in the CTA construction phase, an intense implementation activity is expected within the DATA project. Some major technical choices will be required and the computing model could also evolve in consideration of the CTA sites final location, new requirements and the potential evolution of computing architectures.

\section{Acknowledgments}

We gratefully acknowledge support from the agencies and organisations listed at the following URL: http://www.cta- observatory.org/?q=node/22

\section{References}

[1] B.S. Acharya et al., 2013, Astroparticle Physics 43, 3.

[2] A. Bulgarelli, V. Fioretti, 2015, The On-Site Analysis of the CTA, in these proceedings.

[3] J. Knödlseder et al., 2015, Observer Access to CTA, in these proceedings.

[4] L. Arrabito et al., 2015, CHEP proceedings, 455. 